ISSN 0103-9954

\title{
QUINTAIS AGROFLORESTAIS: ESTRUTURA, COMPOSIÇÃO FLORÍSTICA E ASPECTOS SOCIOAMBIENTAIS EM ÁREA DE ASSENTAMENTO RURAL NA AMAZÔNIA BRASILEIRA
}

\author{
HOME GARDENS: STRUCTURE, FLORISTIC COMPOSITION AND ENVIRONMENTAL ASPECTS \\ IN AREA OF RURAL SETTLEMENT IN BRAZIL'S AMAZON FOREST
}

\author{
Larissa Santos de Almeida ${ }^{1}$ João Ricardo Vasconcellos Gama²
}

\begin{abstract}
RESUMO
O levantamento da estrutura, composição florística e aspectos socioambientais dos quintais agroflorestais foi realizado na comunidade Santo Antônio, município de Santarém, Pará, Brasil. O local de estudo compreende 6 QAFs, onde também foram ouvidos os manejadores de cada quintal e mais 2 moradores antigos. Foi amostrado um total de 522 indivíduos compreendendo 90 espécies e 53 famílias botânicas. A família que apresentou maior número de espécies foi Asteraceae. As espécies destinadas à alimentação (32\%) dominaram a área e as mais frequentes foram Persea americana, Psidium guajava, Carica papaya e Allium fistulosum. A maioria das espécies foi introduzida (78\%), com predominância dos subarbustos (40\%) e árvores (22\%). O manejo dos quintais é realizado pela mulher, responsável pela introdução de novas espécies e sua diversificação tende a evoluir ao longo do tempo. A análise do Coeficiente de Importância da Espécie (CIE) permitiu indicar como potencialmente comerciais Euterpe oleracea, Annona muricata, Carica papaya, Psidium guajava e Carapa guianensis, cujo manejo deve ser favorecido dentro dos quintais, podendo haver sua introdução em lotes, otimizando o uso da terra, assegurando a segurança alimentar e agregando renda às famílias com a venda do excedente da produção.
\end{abstract}

Palavras-chave: assentamento; agricultura familiar; Amazônia.

\begin{abstract}
The structure, floristic and environmental aspects survey of home gardens was performed in the community of Santo Antonio, municipality of Santarém, Pará state, Brazil. The area studied comprises 6 home gardens, where each yard handler and 2 more former residents were heard. A total of 522 plants were sampled comprising 90 species distributed in 53 families. The family with the greatest number of species was Asteraceae. The species for food (32\%) dominated the area and the most frequent ones were Persea americana, Psidium guajava, Carica papaya and Allium fistulosum. Most of species were imported plants (78\%), predominantly subshrubs $(40 \%)$ and trees $(22 \%)$. The management of the gardens is done by a woman who is also responsible for the introduction of new species and its diversification tends to evolve over time. The Analysis of the Species Importance Coefficient indicates how these potentially commercial Euterpe oleracea, Annona muricata, Carica papaya, Psidium guajava and Carapa guianensis, which management should be favored in the agroforestry for example, optimizing the use of land, food security and aggregating income families through the sale of surplus.
\end{abstract}

Keywords: settlement; familiar agriculture; Amazon forest.

1 Engenheira Florestal, Msc., Doutoranda do Programa de Pós-Graduação em Ciências Florestais, Instituto de Ciências Agrárias, Universidade Federal Rural da Amazônia, Av. Presidente Tancredo Neves, 2501, Montese, CEP 66077-901, Belém (PA), Brasil. Bolsista CNPq. larissaflorestal@yahoo.com.br

2 Engenheiro Florestal, Dr., Professor do Instituto de Biodiversidade e Florestas, Universidade Federal do Oeste do Pará, Rua Vera Paz, s/n, Salé, CEP 68135-110, Santarém (PA), Brasil. jrvgama@gmail.com

Recebido para publicação em 20/08/2010 e aceito em 22/05/2013 


\section{INTRODUÇÃO}

O quintal agroflorestal (QAF) é um sistema tradicional de uso da terra amplamente empregado nas regiões tropicais. É um sistema de produção praticado por famílias que vivem em zonas rurais, periurbanas e urbanas, classificado como sistema agroflorestal (SAF), implantado nas áreas contíguas às residências, ou seja, no quintal. Os QAFs são compostos por várias espécies agrícolas e florestais, onde são criados pequenos animais domésticos ou domesticados (CONSTANTIN, 2005; SABLAYROLLES e ANDRADE, 2009).

Os QAFs possuem papel importante na vida das famílias, ressaltadas as de baixa renda, sejam elas rurais ou urbanas, em razão de propiciarem beneficios sociais: recursos terapêuticos, conforto ambiental e recreação para a família; benefícios ecológicos: conservação de material genético in situ e on farm, estabilidade do solo e ciclagem de nutrientes; e benefícios econômicos: geração de receitas extras por meio da venda de frutas em mercados locais (BENTES-GAMA et al., 1999). Almeida e Gama (2010), em prévia avaliação socioeconômica na Comunidade Santo Antônio, concluíram que a área de estudo é carente de serviços de saúde e é distante dos centros urbanos. É importante criar soluções locais para contornar carências socioeconômicas, a começar pela geração de informações iniciais para posteriormente introduzir os QAFs na geração de renda e consolidação da segurança alimentar. Componentes alimento-condimentares e medicinais podem integrar a dieta dos comunitários, representando um fator de contribuição à segurança alimentar local, além de solucionarem os problemas básicos de saúde.

O QAF também constitui um espaço para a convivência, manutenção e a troca de saberes e de espécies (DUBOIS et al., 1996; WINKLERPRINS, 2002) representando, assim, um importante papel socioambiental (ALMEIDA e GAMA, 2010). Diversos trabalhos realizados na Amazônia, principalmente no Estado do Amazonas, destacam os benefícios dos QAFs em áreas ribeirinhas, a saber: aumento da quantidade de alimentos durante o ano, possibilidade de venda do excedente da produção, fortalecimento das relações intra e extrafamiliares, e perpetuação da cultura local (AGUIAR et al., 2009; CASTRO et al., 2009; PINTO et al., 2006; COSTA e MITJA, 2010).

Características destes sistemas de uso da terra em projetos de assentamento do Instituto
Nacional de Colonização e Reforma Agrária (INCRA) são desconhecidos no Estado do Pará. A região estudada é caracterizada pela exploração madeireira e expansão de campos de monocultivos, por esta razão, deve-se garantir que as famílias que ocupam estas áreas estabeleçam uma convivência sustentável com o meio natural. Tais estudos podem identificar potencialidades locais a serem exploradas economicamente, demonstrando a importância dos QAFs como meio de subsistência, de promoção da segurança alimentar e de otimização do uso da terra, que não presumam o desmatamento e o uso agrícola excessivos. A manutenção de germoplasmas on farm nos QAFS enquanto bancos de germoplasmas nativos e o estabelecimento de metodologias de valoração e manejo destas áreas, também devem ser fomentados. Este estudo avaliou a estrutura, composição florística e características socioambientais de quintais da comunidade Santo Antônio, de forma demonstrativa para as demais comunidades rurais da região, que vêm sofrendo com a limitação das atividades econômicas e de subsistência na região.

\section{MATERIAL E MÉTODOS}

\section{Caracterização da área}

A comunidade Santo Antônio ( $3^{\circ} 32$ '58.89'S e 54² $43^{\prime} 57.11^{\prime \prime}$ W) foi fundada no ano 2000 , com a implantação do Projeto de Assentamento (PA) Moju I e II, que faz parte de um programa de ocupação dirigida do Instituto Nacional de Colonização e Reforma Agrária-INCRA, em área de floresta nativa, que deu lugar à área atual do assentamento. É formada por 56 pequenas propriedades rurais que ocupam uma área de, aproximadamente, 5.012,25 ha. Existem famílias que moram em seus lotes (34) e famílias que, apesar de possuírem um lote, residem na agrovila (22). Dentre os lotes, 39 possuem áreas destinadas para desmatamento e reserva legal, e, apenas 17 possuem, além desses usos da terra, área de preservação permanente. A área média dos lotes é de 86,5 ha, sendo que, em torno de 17,17 h, são destinados ao desmatamento legalizado e 67,29 ha à reserva legal. As duas principais formas de obtenção de renda pelos assentados são: i) a venda da madeira retirada dos lotes via manejo florestal na reserva legal por meio de uma parceria do tipo empresa e comunidade, ou por meio de desmatamento autorizado e; ii) a agricultura familiar, realizada na área do lote correspondente à área de uso alternativo 
do solo, cuja extensão máxima na região amazônica equivale, de acordo com a legislação, a $20 \%$ da área.

$\mathrm{O}$ acesso à área da comunidade é realizado exclusivamente via terrestre. Por esta razão, a área do assentamento recebe influência direta da BR 163, que liga Santarém-PA à Cuiabá-MT. A ligação da BR 163 à Rodovia Transamazônica, na região Sul do Estado do Pará possibilitou a imigração de nordestinos, que compõem parte da população do assentamento, assim como, também é composta discretamente por imigrantes da região Sul do Brasil.

A área estudada localiza-se na mesorregião do Baixo Amazonas e na microrregião de Santarém (Figura 1). A comunidade é ligada por uma estrada não pavimentada (vicinal) à rodovia BR 163, cuja extensão é de, aproximadamente, $23 \mathrm{~km}$. O clima é o tropical úmido, possui variação térmica anual inferior a $5^{\circ} \mathrm{C}$ e temperatura média anual de $25,5^{\circ} \mathrm{C}$, temperaturas médias do mês mais frio sempre superiores a $18^{\circ} \mathrm{C}$, umidade relativa média do ar de $88 \%$ e precipitação anual média de $1820 \mathrm{~mm}$. O regime de chuvas apresenta grande variação durante o ano, com as maiores elevações nos meses de janeiro a maio e a estação seca de agosto a novembro. A altitude na área de estudo é de aproximadamente $170 \mathrm{~m}$ (RODRIGUES, 2001). Os solos predominantes são os latossolos amarelo e vermelho-amarelo, com a presença de uma camada de argila caulinítica arenosa, de média a alta plasticidade, com uma espessura entre $10 \mathrm{e}$ 20 m (IBGE, 1992). A vegetação característica da região é do tipo Floresta Ombrófila Densa de terra firme (VELOSO et al., 1991). O dossel é denso, fechado e compacto, situado entre 30 e $35 \mathrm{~m}$ de altura, interceptando grande parte da energia solar, passando somente uma pequena fração, em torno de
$5 \%$, que chega até o chão da floresta (SALOMÃO et al., 2007).

\section{Coleta e análise dos dados}

Considerando que a comunidade foi fundada no ano de 2000, uma avaliação visual permitiu o enquadramento dos QAFs em novos (idade- $i<5$ anos), intermediários ( 5 anos $\leq i<8$ anos) e antigos ( $\mathrm{i} \geq 8$ anos) (o que representou uma amostra de aproximadamente $27 \%$ dos QAFs da agrovila). Em cada categoria, foram incluídos dois quintais, nos quais foram feitas análises da estrutura, da composição florística e das características socioambientais, sendo este último aspecto avaliado por meio da aplicação de questionários. As entrevistas foram conduzidas junto ao componente do grupo familiar presente capaz de informar sobre o QAF. Os questionários foram classificados como estruturados e o método utilizado adicionalmente foi a observação direta, conforme as metodologias de Goode e Hatt (1969) e Lakatos e Marconi (2001).

Além da aplicação de seis questionários por QAF, realizaram-se mais duas entrevistas propositais com moradores residentes na agrovila desde sua implantação que foram entrevistados com o objetivo de embasar as informações gerais da implantação da comunidade no assentamento. Posteriormente, foi selecionado um quintal agroflorestal para observar a extensão e a tendência de disposição dos elementos nos QAFs, aspectos visualmente similares entre todos os quintais da agrovila. Foi observada a presença de criações. Todas as espécies vegetais foram inventariadas anotando-se o nome regional. Foram anotados, ainda, forma de vida das plantas e seus usos. Conforme Bentes-Gama et al. (1999), as espécies foram analisadas por meio do Coeficiente

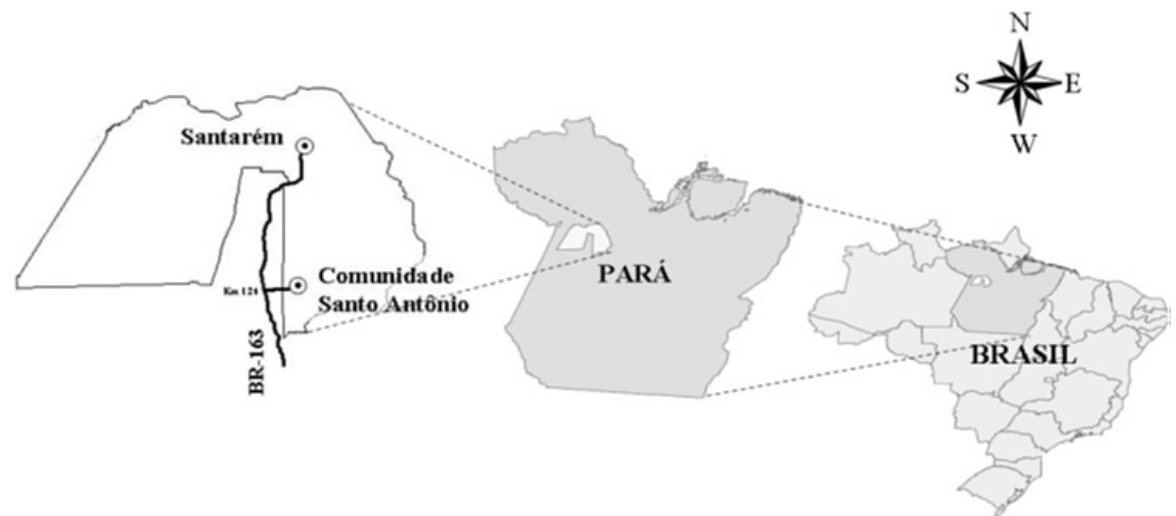

FIGURA 1: Comunidade Santo Antônio, Assentamento Moju I e II, BR 163, Santarém, Pará. FIGURE 1: Santo Antonio community, Moju I and II Settlement, BR 163 Highway, Santarém, Pará. 
de Importância da Espécie (CIE):

$$
C I E=\frac{3 \cdot N U+2 \cdot I B+D C}{6}
$$

Em que: Nível de Utilização $(\mathrm{NU})=$ expressa a importância da espécie quanto a sua funcionalidade para a família: 3 - muito utilizada (espécie com três ou mais usos); 2 - utilizada (espécie com dois usos); 1 - pouco utilizada (espécie com um único uso).

Importância Biofísica (IB) = representa a ocorrência das espécies: $\quad 3$ - alta freqüência de 70 a $100 \%$ ); 2 - média (frequência de 31 a $69 \%$ ); 1 - baixa (frequência igual ou menor do que $30 \%$ ).

Demanda de Comercialização (DC) = está relacionada ao potencial de comercialização da espécie: 3 - alta (muito demandada); 2 - média (mediamente demandada); 1 - baixa (pouco demandada) e 0 -inexistente.

\section{RESULTADOS E DISCUSSÃO}

\section{Aspectos socioeconômicos}

A comunidade possui uma escola de ensino fundamental que atende à demanda local e de oito comunidades vizinhas e oferta o programa de educação de jovens e adultos no período noturno. Não há atendimento em saúde no local e os hospitais mais próximos estão localizados nos centros urbanos de Belterra e Santarém, distantes, aproximadamente, 100 e $157 \mathrm{~km}$, respectivamente, que atendem à demanda da comunidade nos casos mais graves. Os problemas menos graves de saúde relatados são febres, gripes e verminoses, sendo que este último é decorrente da precariedade da rede sanitária.

A água consumida na comunidade provém de um microssistema ao custo de R \$ 5,00 mês ${ }^{-1}$ família ${ }^{-1}$. O meio de comunicação predominante é a televisão, além da carta ou bilhete enviado via ônibus. O trecho da linha de ônibus liga a comunidade à Santarém duas vezes por semana, ao custo de R\$ 14,00 , da qual depende o escoamento da produção familiar e o deslocamento dos comunitários. A única produção em escala comercial é de Piper nigrum L. (pimenta-do-reino) e os cultivos de subsistência são de Oriza sp. (arroz), Phaseolus vulgaris (feijão), Zea sp. (milho) e Manihot sp. (mandioca).

\section{Manejo}

A maior parte do trabalho nos quintais é desenvolvida pela mulher, com raro auxílio dos demais membros da família. Deste modo, a mulher representa força de trabalho na unidade familiar, pois além das atividades produtivas, ainda é responsável pelas tarefas domiciliares. A predominância da mulher no cultivo e manejo dos QAFs assemelha-se aos resultados de Vieira (2006), que concluiu que as mulheres são as responsáveis pela implantação e manejo dos quintais nos municípios de Benevides e Igarapé-açu no Estado do Pará. Segundo Rosa et al. (2007), a divisão de trabalho é uma estratégia dos agricultores para aumentar a eficiência da mão de obra familiar. Na Reserva Extrativista Tapajós ArapiunsPA, Ferreira e Sablayrolles (2009) concluíram que o papel da mulher nos quintais é predominante, visto que, dos 20 quintais pesquisados, 17 são manejados exclusivamente por mulheres. Tal como encontraram Vieira et al. (2012), em Bonito, Pará, há pouca tecnologia empregada nos quintais.

\section{Inventário das espécies nos quintais}

Os QAFs apresentaram uma área média de $720 \mathrm{~m}^{2}$. Ao todo, foram amostrados 522 indivíduos distribuídos em 90 espécies e 53 famílias botânicas. O número de indivíduos e espécies por quintal variou de acordo com a idade dos QAFs (Figura 2). Nos quintais antigos ( $\geq 8$ anos) registrou-se, em média, 133 indivíduos e 43 espécies por quintal. Nos quintais intermediários (5 anos $\leq \mathrm{i}<8$ anos) ocorreram em média 77 indivíduos pertencentes a 32 espécies por quintal. Os quintais mais recentes ( $i<5$ anos) incluíram os menores valores da amostra: em média 51 indivíduos pertencentes a 15 espécies por QAF.

A família mais importante na amostra foi Asteraceae (seis espécies), seguida por Anacardiaceae e Solanaceae (quatro), Annonaceae, Araceae Lamiaceae, Malvaceae, Moraceae, Rutaceae e Verbenaceae (três espécies cada) (Tabela 1). As famílias botânicas mais bem representadas no levantamento de Santos (2006) em QAFs de áreas ribeirinhas na região do rio Sucuriju e região dos lagos no Amapá foram Solanaceae e Lamiaceae. Lunz (2007), analisando a estrutura de QAFs em 30 lotes de assentados em Nova Califórnia (BR 364), Rondônia, registrou 155 espécies pertencentes a 74 famílias botânicas, com destaque para as famílias Myrtaceae e Arecaceae. 


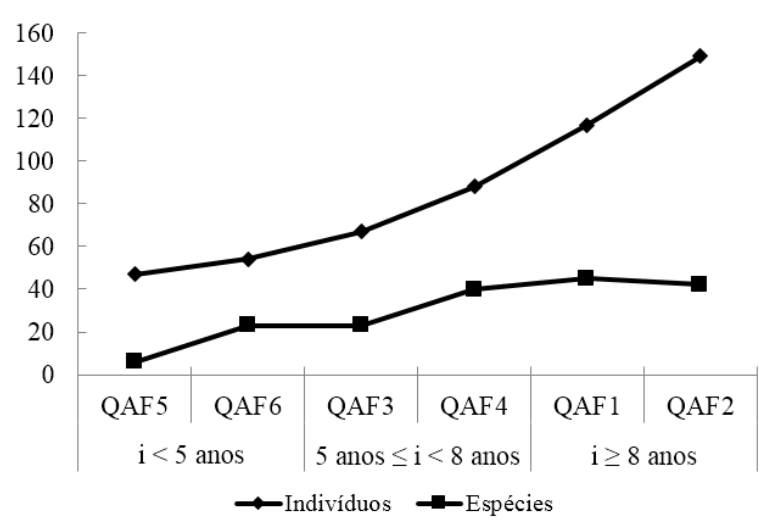

FIGURA 2: Número de indivíduos e espécies em quintais agroflorestais de diferentes idades, comunidade Santo Antônio, Assentamento Moju I e II, BR 163, Santarém, Pará.

FIGURE 2: Number of individuals and species in home gardens of different ages, Santo Antonio community, Moju I and II Settlement, BR 163 Highway, Santarém, Pará state.

A maior parte das espécies cultivadas nos QAFs é destinada à alimentação das famílias. No que se refere à ocorrência dessas espécies, dentre as quatro presentes em todos os quintais $(100 \%$ de frequência), destacam-se Persea americana (abacate), Psidium guajava (goiaba) e Carica papaya (mamão) cujos frutos são apreciados pelos comunitários; e Allium fistulosum (cebolinha) por ser muito utilizada como condimento na culinária regional. Espécies como Cichorium sp. e Cichorium intybus L., que em outras regiões são consideradas apenas verduras, são determinadas como condimentos na comunidade.

Quanto à importância das espécies, considerando o CIE, a espécie mais importante foi Mangifera indica (manga), devido ao fato da espécie apresentar alta produção de frutos, ser muito consumida pelas famílias e ocorrer em $83,3 \%$ dos quintais, embora a demanda de comercialização seja inexistente na comunidade. Os frutos de Mangifera indica são matéria-prima de xaropes para gripe (Tabela 1). Psidium guajava foi a segunda espécie mais importante.

Bentes-Gama et al. (1999), estudando a estrutura e a composição florística dos QAFs em Bragança, nordeste paraense, também atribuíram à Mangifera indica o maior valor para o CIE e Psidium guajava obteve $100 \%$ de frequência nos
QAFs avaliados pelos autores.

As demais espécies que também se destacaram foram: Cocus nucifera (coco), Annona muricata (graviola), Carica papaya (mamão), Citrus limonia (limão), sendo utilizadas na alimentação das famílias e com ocorrência nos QAFs. A palmeira Euterpe oleracea (açaí), destacou-se por sua alta demanda de comercialização. Jatropha gossypiifolia (pião-roxo) e Carapa guianensis (andiroba) são consideradas importantes do ponto de vista medicinal no preparo de chás e para o uso do óleo, respectivamente.

Na região Nordeste do País, Albuquerque et al. (2005) estudaram os quintais em Alagoinha e registraram uma alta densidade de espécies frutíferas, como Mangifera indica, Citrus sinensis, Psidium guajava, Anacardium ocidentale e Carica papaya. Nos quintais pesquisados por Semedo e Barbosa (2007), em áreas urbanas de Boa Vista $\mathrm{RO}$, predominaram espécies frutíferas como: Cocus nucifera, Mangifera indica e Syzygium malaccence. Segundo os autores, o cultivo destas espécies está relacionado à preferência pelo sabor e a facilidade de implantação, visto que não há necessidade de tratos culturais específicos, isto é, apresentam maior rusticidade.

Pode-se inferir uma relação entre o valor nutricional das espécies frutíferas e sua ocorrência nos QAFs. Espécies como Cocus nucifera (rico em proteínas) e Mangifera indica (vitamina A), além de Malpighia emarginata (acerola), Anacardium ocidentale (caju), Citrus limonia e Psidium guajava (vitamina C), por exemplo, são encontradas frequentemente nos quintais. Conforme Semedo e Barbosa (2007), mesmo que a inserção de espécies ocorra ao acaso, estas contribuem para a suplementação da dieta das populações locais. É válido ressaltar que no âmbito internacional, a segurança alimentar é preconizada por organismos e entidades, tendo como principal órgão a Organização para as Nações Unidas para a Agricultura (FAO).

A crescente demanda mundial por alimentos pode gerar um avanço sobre as áreas em que é praticada a agricultura familiar, pois as grandes áreas monocultivadas tendem a suprimilas. Na região de influência da BR 163, a fronteira de ocupação agropecuária vem avançando sobre os minifúndios, o que representa uma ameaça aos pequenos produtores. Estes se veem, em muitos casos, deslumbrados pela oferta de dinheiro por suas terras, fato anteriormente nunca visto por eles. Por esta razão, incentivos às práticas de otimização 
TABELA 1: Espécies encontradas nos quintais agroflorestais da comunidade Santo Antônio, BR 163, Santarém, Pará. O (origem); FV (forma de vida); U (uso); NPQ (número médio de plantas por quintal); CIE (coeficiente de importância da espécie).

TABLE 1: List of species found in the home gardens of Santo Antonio community, BR 163 Highway, Santarém, Pará state: Where: O (origin); HE (ecological habit), U (use), NPH (average number of plants by homegarden), CIS (coefficient of importance of the species).

\begin{tabular}{|c|c|c|c|c|c|c|}
\hline Família/Nome Científico & Nome regional & $\mathrm{O}$ & $\mathrm{FV}$ & $\mathrm{U}$ & NPQ & CIE \\
\hline Acanthaceae & & & & & 0,0 & \\
\hline Justicia acuminatissima (Miq.) Bremek & Saratudo & $\mathrm{N}$ & 1 & 4 & 0,3 & 1,3 \\
\hline Alliaceae & & & & & 0,0 & \\
\hline Allium fistulosum $\mathrm{L}$. & Cebolinha & $\mathrm{E}$ & 4 & 5 & 8,8 & 1,7 \\
\hline Amaranthaceae & & & & & 0,0 & \\
\hline Alternanthera brasiliana (L.) Kuntze & Terramicina & E & 7 & 4 & 0,2 & 0,8 \\
\hline Anacardiaceae & & & & & 0,0 & \\
\hline Anacardium ocidentale $\mathrm{L}$. & Caju & $\mathrm{E}$ & 1 & 2 & 1,0 & 1,2 \\
\hline Mangifera indica $\mathrm{L}$. & Manga & $\mathrm{E}$ & 1 & $2 ; 4 ; 7$ & 2,5 & 2,5 \\
\hline Spondias dulcis Parkinson & Cajamanga & $\mathrm{E}$ & 1 & 2 & 0,2 & 0,8 \\
\hline Spondias sp. & Taperebá & $\mathrm{N}$ & 1 & 2 & 0,3 & 1,3 \\
\hline Annonaceae & & & & & 0,0 & \\
\hline Annona squamosa $\mathrm{L}$. & Ata & $\mathrm{E}$ & 2 & 2 & 1,2 & 1,2 \\
\hline Annona muricata $\mathrm{L}$. & Graviola & $\mathrm{E}$ & 1 & $2 ; 4$ & 4,3 & 2,2 \\
\hline Rollinia тисоsa (Jacq.) Baill & Biriba & $\mathrm{N}$ & 1 & 2 & 0,2 & 1,0 \\
\hline Apiaceae & & & & & 0,0 & \\
\hline Coriandrum sativum L. & Coentro & E & 4 & 5 & 0,2 & 1,0 \\
\hline Araceae & & & & & 0,0 & \\
\hline Anthurium sp. & Antúrio & $\mathrm{E}$ & 3 & 3 & 0,2 & 1,3 \\
\hline Caladium sp. & Tajá & $\mathrm{N}$ & 4 & 3 & 5,3 & 1,3 \\
\hline Epipremnum pennatum (L.) Engl. & Jiboia & $\mathrm{E}$ & 3 & 3 & 0,3 & 0,8 \\
\hline Arecaceae & & & & & 0,0 & \\
\hline Cocos nucifera $\mathrm{L}$. & Coco & $\mathrm{E}$ & 5 & $2 ; 3$ & 2,7 & 2,2 \\
\hline Euterpe oleracea Mart. & Açaí & $\mathrm{N}$ & 5 & $2 ; 3$ & 3,0 & 2,2 \\
\hline Asteraceae & & & & & 0,0 & \\
\hline Cichorium sp. & Almeirão & E & 3 & 5 & 0,2 & 0,8 \\
\hline Cichorium intybus $\mathrm{L}$. & Chicória & $\mathrm{E}$ & 4 & $4 ; 5$ & 0,2 & 1,3 \\
\hline Dahlia sp. & Rosa vermelha & $\mathrm{E}$ & 4 & 3 & 0,7 & 1,7 \\
\hline Spilanthes oleraceae L. & Jambu & $\mathrm{N}$ & 7 & $2 ; 4$ & 0,2 & 1,5 \\
\hline Spilanthes acmella $\mathrm{L}$. & Jerimum & $\mathrm{E}$ & 7 & 2 & 0,2 & 1,0 \\
\hline Tagetes minuta $\mathrm{L}$. & Cravo & E & 3 & 3 & 0,2 & 1,3 \\
\hline Brassicaceae & & & & & 0,0 & \\
\hline Brassica sp. & Couve & E & 4 & 2 & 0,5 & 1,0 \\
\hline Bromeliaceae & & & & & 0,0 & \\
\hline Ananas comosus (L.) Merril & Abacaxi & $\mathrm{E}$ & 3 & 2 & 1,8 & 1,7 \\
\hline Indeterminado & Bromélia & - & 3 & 3 & 0,2 & 1,3 \\
\hline Caesalpiniaceae & & & & & 0,0 & \\
\hline Bauhinia forficata $\mathrm{L}$. & Pata-de-vaca & $\mathrm{E}$ & 2 & 4 & 0,3 & 0,8 \\
\hline Caesalpinia ferrea Mart. & Jucá & $\mathrm{E}$ & 2 & 4 & 0,2 & 0,8 \\
\hline
\end{tabular}

Continua... 
TABELA 1: Continuação...

TABLE 1: Continued...

\begin{tabular}{|c|c|c|c|c|c|c|}
\hline Família/Nome Científico & Nome regional & $\mathrm{O}$ & FV & $\mathrm{U}$ & NPQ & CIE \\
\hline Caricaceae & & & & & 0,0 & \\
\hline Carica papaya L. & Mamão & $\mathrm{E}$ & 2 & $2 ; 4$ & 2,7 & 2,2 \\
\hline Caryocaraceae & & & & & 0,0 & \\
\hline Caryocar villosum (Aubl.) Pers. & Piquiá & $\mathrm{N}$ & 1 & $2 ; 4$ & 0,2 & 1,7 \\
\hline Cecropiaceae & & & & & 0,0 & \\
\hline Cecropia sp. & Embaúba & $\mathrm{N}$ & 1 & $1 ; 4$ & 0,3 & 1,3 \\
\hline Compositae & & & & & 0,0 & \\
\hline Dendranthema grandiflora Tzvelev. & Crisântemo & $\mathrm{E}$ & 3 & 3 & 0,8 & 1,3 \\
\hline Compostaceaea & & & & & 0,0 & \\
\hline Ocimum basilicum $\mathrm{L}$. & Manjericão & $\mathrm{E}$ & 4 & $4 ; 5$ & 1,3 & 1,5 \\
\hline Convolvulaceae & & & & & 0,0 & \\
\hline Ipomea batatas (L.) Lam. & Batata-doce & $\mathrm{E}$ & 7 & 2 & 0,2 & 1,2 \\
\hline Crassulaceae & & & & & 0,0 & \\
\hline Kalanchoe brasiliensis Camb. & Coramina & $\mathrm{E}$ & 3 & 4 & 1,2 & 1,2 \\
\hline Curcubitaceae & & & & & 0,0 & \\
\hline Luffa operculata (L.) Cogn & Bucha & $\mathrm{N}$ & 3 & $4 ; 7$ & 0,2 & 1,5 \\
\hline Dioscoreaceae & & & & & 0,0 & \\
\hline Dioscorea alata $\mathrm{L}$. & Cará & $\mathrm{N}$ & 7 & $2 ; 4$ & 0,2 & 1,7 \\
\hline Euphorbiaceae & & & & & 0,0 & \\
\hline Jatropha gossypiifolia $\mathrm{L}$. & Pião-roxo & $\mathrm{E}$ & 3 & $3 ; 4 ; 6$ & 1,5 & 2,2 \\
\hline Manihot sp. & Macaxeira & $\mathrm{E}$ & 3 & 2 & 0,5 & 1,3 \\
\hline Fabaceae & & & & & 0,0 & \\
\hline Dipteryx odorata (Aubl.) Willd. & Cumaru & $\mathrm{N}$ & 1 & 4 & 0,2 & 1,3 \\
\hline Erythrina variegata Merr. & Brasileirinho & $\mathrm{E}$ & 1 & 3 & 0,5 & 0,8 \\
\hline Lamiaceae & & & & & 0,0 & \\
\hline Coleus barbatus Benth. & Melhoral & $\mathrm{E}$ & 3 & 4 & 0,3 & 1,2 \\
\hline Ocimum selloi Benth. & Elixir paregórico & $\mathrm{E}$ & 3 & 4 & 0,3 & 1,2 \\
\hline Plectranthus amboinicus (Lour.) Spreng. & Folha-grossa & $\mathrm{E}$ & 3 & 4 & 0,3 & 1,2 \\
\hline Lauraceae & & & & & 0,0 & \\
\hline Persea americana Mill. & Abacate & $\mathrm{E}$ & 1 & 2 & 3,7 & 2,0 \\
\hline Liliaceae & & & & & 0,0 & \\
\hline Sansevieria zeylanica Willd. & Espada-de-são-jorge & $\mathrm{E}$ & 3 & $3 ; 6$ & 1,0 & 1,7 \\
\hline Malpighiaceae & & & & & 0,0 & \\
\hline Byrsonima crassifólia & Muruci & $\mathrm{E}$ & 1 & $2 ; 4$ & 0,2 & 1,7 \\
\hline Malpighia emarginata L. & Acerola & $\mathrm{E}$ & 2 & $2 ; 3$ & 1,2 & 2,0 \\
\hline Malvaceae & & & & & 0,0 & \\
\hline Gossypium sp. & Algodão-branco & $\mathrm{E}$ & 2 & 7 & 0,2 & 0,8 \\
\hline Hibiscus esulentus L. & Quiabo & $\mathrm{E}$ & 2 & $2 ; 4$ & 0,2 & 1,7 \\
\hline Hibiscus rosa-sinensis L. & Papoula & $\mathrm{E}$ & 2 & 3 & 2,3 & 1,2 \\
\hline Meliaceae & & & & & 0,0 & \\
\hline Carapa guianensis Aubl. & Andiroba & $\mathrm{N}$ & 1 & $1 ; 4$ & 2,8 & 2,2 \\
\hline Mimosaceae & & & & & 0,0 & \\
\hline Inga sp. & Ingá & $\mathrm{N}$ & 1 & 2 & 0,5 & 1,2 \\
\hline
\end{tabular}

Continua... 
TABELA 1: Continuação...

TABLE 1: Continued...

\begin{tabular}{|c|c|c|c|c|c|c|}
\hline Família/Nome Científico & Nome regional & $\mathrm{O}$ & FV & $\mathrm{U}$ & NPQ & CIE \\
\hline Moraceae & & & & & 0,0 & \\
\hline Artocarpus heterophylla Lam. & Jaca & $\mathrm{E}$ & 1 & 2 & 0,8 & 1,5 \\
\hline Ficus sp. & Ficus & $\mathrm{E}$ & 2 & 3 & 0,3 & 0,8 \\
\hline Morus alba L. & Amora & $\mathrm{E}$ & 2 & 2 & 2,0 & 1,2 \\
\hline Musaceae & & & & & 0,0 & \\
\hline Musa sp. & Banana & $\mathrm{E}$ & 2 & 2 & 0,8 & 1,7 \\
\hline Myrtaceae & & & & & 0,0 & \\
\hline Eugenia sp. & Jambo & $\mathrm{E}$ & 1 & 2 & 0,5 & 0,8 \\
\hline Psidium guajava $\mathrm{L}$. & Goiaba & $\mathrm{E}$ & 1 & $2 ; 4$ & 8,2 & 2,3 \\
\hline Nyctaginaceae & & & & & 0,0 & \\
\hline Mirabilis jalapa $\mathrm{L}$. & Maravilha & $\mathrm{E}$ & 2 & $3 ; 4$ & 0,2 & 1,8 \\
\hline Orchidaceae & & & & & 0,0 & \\
\hline Indeterminado & Orquídea-branca & $\mathrm{N}$ & 3 & 3 & 0,3 & 1,3 \\
\hline Indeterminado & Orquídea-capim & $\mathrm{N}$ & 3 & 3 & 0,3 & 1,3 \\
\hline Palmae & & & & & 0,0 & \\
\hline Oenocarpus bacaba Mart. & Bacaba & $\mathrm{N}$ & 5 & 2 & 0,2 & 1,2 \\
\hline Syagrus pseudococos (Raddi) Glassman & Piririma & $\mathrm{N}$ & 5 & 4 & 0,2 & 0,8 \\
\hline Passifloraceae & & & & & 0,0 & \\
\hline Passiflora edulis Sims & Maracujá & $\mathrm{E}$ & 7 & $2 ; 4$ & 0,2 & 1,3 \\
\hline Piperaceae & & & & & 0,0 & \\
\hline Ottonia corcovadensis Miq. & Jaborandi & $\mathrm{E}$ & 3 & 4 & 0,2 & 0,8 \\
\hline Piper nigrum $\mathrm{L}$. & Pimenta-do-reino & $\mathrm{E}$ & 7 & 5 & 0,2 & 1,3 \\
\hline Poaceae & & & & & 0,0 & \\
\hline Cymbopogon citratus $\mathrm{L}$. & Capim-santo & $\mathrm{E}$ & 4 & 4 & 0,2 & 0,8 \\
\hline Zea mays $\mathrm{L}$. & Milho & $\mathrm{E}$ & 4 & 2 & 0,3 & 1,3 \\
\hline Punicaceae & & & & & 0,0 & \\
\hline Punica granatum $\mathrm{L}$. & Romã & $\mathrm{E}$ & 3 & 4 & 0,2 & 1,0 \\
\hline Rosaceae & & & & & 0,0 & \\
\hline Cydonia vulgaris $\mathrm{T}$. & Marmelo & $\mathrm{E}$ & 3 & 2 & 0,2 & 0,8 \\
\hline Rubiaceae & & & & & 0,0 & \\
\hline Coffea arábica $\mathrm{L}$. & Café & $\mathrm{E}$ & 3 & 2 & 1,3 & 1,2 \\
\hline Ixora sp. & Ixora & $\mathrm{E}$ & 3 & 3 & 0,7 & 1,2 \\
\hline Rutaceae & & & & & 0,0 & \\
\hline Citrus limonia Osbeck & Limoeiro & $\mathrm{E}$ & 2 & $2 ; 4$ & 1,3 & 2,2 \\
\hline Citrus sinensis (L.) Osbeck & Laranja & $\mathrm{E}$ & 2 & $2 ; 4$ & 1,7 & 1,8 \\
\hline Ruta graveolens L. & Arruda & $\mathrm{E}$ & 3 & $4 ; 6$ & 0,2 & 1,3 \\
\hline Salviniaceae & & & & & 0,0 & \\
\hline Salvinia $s p$ & Marrequinha & $\mathrm{E}$ & 3 & 3 & 0,2 & 0,8 \\
\hline Sapindaceae & & & & & 0,0 & \\
\hline Talisia esculenta Radlk. & Pitomba & $\mathrm{N}$ & 1 & 2 & 0,8 & 1,2 \\
\hline Sapotaceae & & & & & 0,0 & \\
\hline Pouteria speciosa (Ducke) Baehni & Pajurá & $\mathrm{N}$ & 1 & 2 & 0,2 & 1,0 \\
\hline Scrophulariaceae & & & & & 0,0 & \\
\hline
\end{tabular}

Continua...

Ci. Fl., v. 24, n. 4, out.-dez., 2014 
TABELA 1: Continuação...

TABLE 1: Continued...

\begin{tabular}{|c|c|c|c|c|c|c|}
\hline Família/Nome Científico & Nome regional & $\mathrm{O}$ & FV & $\mathrm{U}$ & NPQ & CIE \\
\hline Bacopa sp. & Hortelã & $\mathrm{E}$ & 3 & 4 & 0,2 & 0,8 \\
\hline Solanaceae & & & & & 0,0 & \\
\hline Capsicum annum $\mathrm{L}$. & Pimentão & $\mathrm{E}$ & 3 & $4 ; 6$ & 0,8 & 1,5 \\
\hline Capsicum sp. & Pimenta-malagueta & $\mathrm{E}$ & 3 & 5 & 0,2 & 1,0 \\
\hline Datura suaveolens H. et B. ex Willd. & Saia-branca & $\mathrm{E}$ & 3 & 3 & 0,7 & 1,3 \\
\hline Lycopersicum esculentum Mill. & Tomate & $\mathrm{E}$ & 3 & 2 & 0,2 & 1,3 \\
\hline Sterculiaceae & & & & & 0,0 & \\
\hline Theobroma grandiflorum (Spreng.) Schum. & Cupuaçu & $\mathrm{N}$ & 1 & 4 & 3,2 & 2,0 \\
\hline Verbenaceae & & & & & 0,0 & \\
\hline Duranta repens $\mathrm{L}$. & Pingo-de-ouro & $\mathrm{E}$ & 3 & 3 & 0,3 & 1,3 \\
\hline Duranta sp. & Duranta & $\mathrm{E}$ & 3 & 3 & 0,2 & 0,8 \\
\hline Lantana camara $L$. & Chumbinho & $\mathrm{E}$ & 3 & 3 & 0,2 & 0,8 \\
\hline Vitaceae & & & & & 0,0 & \\
\hline Vitis sp. & Uva & $\mathrm{E}$ & 7 & 2 & 0,2 & 0,8 \\
\hline Zingiberaceae & & & & & 0,0 & \\
\hline Costus spicatus (Jacq.) Sw. & Cana-mansa & $\mathrm{N}$ & 3 & 4 & 0,3 & 1,2 \\
\hline Zingiber officinale $\mathrm{L}$. & Gengibre & $\mathrm{E}$ & 4 & 4 & 0,3 & 1,3 \\
\hline NI 1 & & & & & 0,0 & \\
\hline Indeterminado & NI 1 & $\mathrm{E}$ & 3 & 3 & 0,2 & 0,8 \\
\hline NI 2 & & & & & 0,0 & \\
\hline Indeterminado & NI 2 & $\mathrm{E}$ & 3 & 3 & 0,3 & 0,8 \\
\hline NI 3 & & & & & 0,0 & \\
\hline Indeterminado & NI 3 & $\mathrm{E}$ & 3 & 3 & 0,2 & 0,8 \\
\hline NI 4 & & & & & 0,0 & \\
\hline Indeterminado & $N I 4$ & $\mathrm{E}$ & 3 & 3 & 0,2 & 0,8 \\
\hline NI 5 & & & & & 0,0 & \\
\hline Indeterminado & NI 5 & $\mathrm{E}$ & 3 & 3 & 0,2 & 0,8 \\
\hline TOTAL & & & & & 87,0 & \\
\hline
\end{tabular}

Em que: $\mathrm{O}=$ Origem, (N) Nativa, (E) Exótica; FV = Forma de Vida, (1) Arbóreo, (2) Arbusto, (3) Subarbusto, (4) Herbáceo, (5) Palmeira, (6) Rastejante, (7) Trepadeira; U = Usos, (1) Madeira; (2) Alimentação; (3) Ornamental; (4) Medicinal; (5) Condimento; (6) Místico; (7) Outro.

da unidade de produção familiar podem impedir o avanço sobre as pequenas propriedades. A garantia de subsistência e, se possível, a venda do excedente da produção contribui para a permanência das famílias de forma digna no meio rural, bem como inibe problemas urbanos, causados, por exemplo, pelo êxodo das famílias do campo.

A distância da comunidade dos centros urbanos fundamenta a inserção de diferentes espécies (Tabela 1), não apenas de frutíferas, como também de espécies medicinais, entre outros usos. Assim, são delineadas as funções social, econômica e ecológica dos QAFs para a comunidade Santo Antônio.

Predominaram espécies exóticas, que perfizeram $78 \%$ do total. Este resultado condiz com a crescente influência externa decorrente, principalmente, de aspectos como a miscigenação de culturas, por sua vez justificada pela imigração. As espécies nativas ocorreram em todos os quintais, resultado comum em estudos em outras regiões (RICO-GRAY et al., 1990; NAIR, 2004; ALBUQUERQUE et al., 2005). Semedo e Barbosa (2007) comentam que a migração contribui culturalmente com a 
incorporação de informações sobre o uso dos recursos regionais. Assim, as variedades inexistentes em determinada área (plantas exóticas) são incorporadas aos hábitos locais. Florentino et al. (2007) encontraram elevado percentual de espécies exóticas na localidade por eles estudada, na maioria dos casos, trazidas por parentes, amigos ou vizinhos.

As espécies nativas perfizeram 22\%. Um exemplo é a ocorrência de Cecropia sp., demonstrando que o banco de sementes do solo influenciou na presença de espécies nos QAFs, já que não houve decisão de cultivo do manejador do quintal, existindo, neste caso, um elemento espontâneo.

Espécies arbóreas em geral dominam os quintais agroflorestais na Amazônia (BENTESGAMA et al., 1999; LOURENÇO et al., 2009; COSTA e MITJA, 2010; SABLAYROLLES e ANDRADE, 2009). Na comunidade, os quintais mais antigos foram compostos por mais árvores. Nos quintais mais jovens foram amostradas mais árvores com pequenos diâmetros, ou seja, ocorreram mais indivíduos nas menores classes de tamanho
(1, 2 e 3), também predominaram os subarbustos (40\%), seguidos das espécies arbóreas (22\%), do tipo arbusto $(15 \%)$, herbáceo $(11 \%)$, rastejante $(5 \%)$, palmeira (4\%) e trepadeira (3\%) (Figura 3$)$.

Quanto à utilização das espécies dos quintais, predominaram aquelas destinadas ao uso alimentar, que totalizaram 39 espécies (32\%) (Figura 2). Sablayrolles e Andrade (2009) analisaram 11 quintais agroflorestais e sua importância para comunidades ribeirinhas em Aveiro-PA, e atribuíram o uso alimentar à maioria das espécies cultivadas, assim como concluíram Rosa et al. (2007) em quintais na Zona Bragantina, Estado do Pará e Rondon-Neto et al. (2004) no Assentamento Rural Teixeira Soares, Estado do Paraná, que, embora em outra região, apresenta características similares.

Trinta e oito espécies possuem uso medicinal $(31,1 \%)$, o que remete à carência de assistência em saúde descrita no levantamento socioeconômico, fato comum às comunidades amazônicas isoladas. Lunz (2007) esclarece que o uso de espécies cultivadas

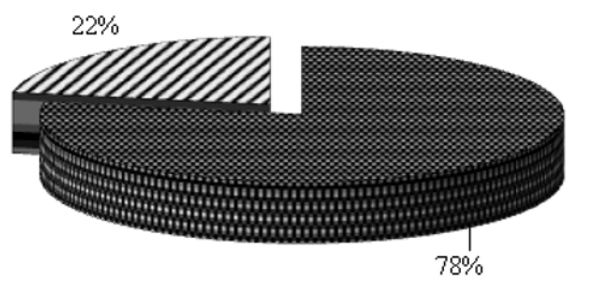

a) 圆Exótica $\square$ Nativa

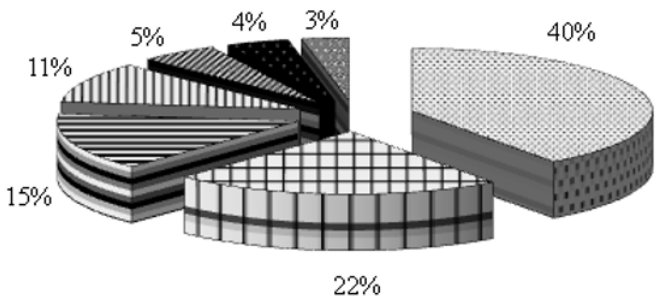

b)

항 subarbusto 日arbóreo Earbusto mherbáceo Zrastejante - palmeira mtrepadeira c)

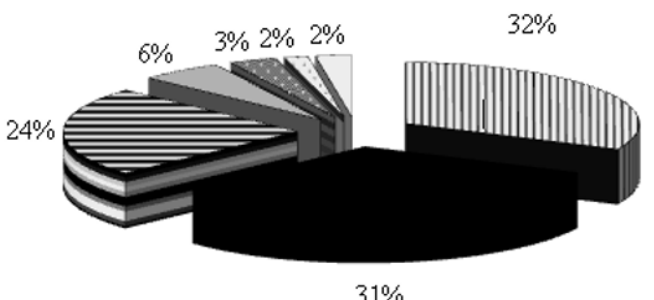

๓Alimentação

- Medicinal

EOrnamental

$\square$ Condimento

Místico

口Madeira

口Outros usos

FIGURA 3: Origem (a), forma de vida (b) e uso das espécies (c) encontradas nos quintais agroflorestais da comunidade Santo Antônio, Assentamento Moju I e II, BR 163, Santarém, Pará.

FIGURE 3: Source (a), ecological habit (b) and use of species (c) found in the home gardens of Santo Antonio community, Moju I and II Settlement, BR 163 Highway, Santarém, Pará state. 
nos QAFs no tratamento de doenças é de suma importância para a comunidade, tanto pela pouca assistência médica no local, quanto pelo alto preço dos medicamentos industrializados, o que contribui para o uso de plantas. A presença destas nos QAFs pesquisados com nomes vernaculares de remédios conhecidos como "melhoral" e "elixir paregórico" reforça a importância destes quanto ao aspecto saúde, substituindo aqueles industrializados.

As espécies ornamentais (29) totalizaram $23,8 \%$ do povoamento, seguidas pelas condimentares com sete espécies $(5,7 \%)$, quatro com significado místico $(3,3 \%)$, duas com potencial madeireiro e três utilizadas para outros fins $(2,5 \%)$. Vale considerar que uma mesma espécie apresentou mais de uma indicação (Tabela 1 e Figura 3).

Dentre as espécies de uso múltiplo, duas possuem três usos: Mangifera indica no aproveitamento dos frutos na alimentação, melhoria do microclima (conforto ambiental) e uso medicinal (xarope da casca fresca indicado para gripe); e Jatropha gossypiifolia (pião-roxo), no uso ornamental, místico contra "mau-olhado" e medicinal (exudato das folhas frescas como cicatrizante e anti-inflamatório).

A disposição das espécies nos quintais não segue um padrão definido, embora algumas espécies de grande porte sejam mantidas distantes da residência. Há espécies dominadas, principalmente nos quintais intermediários e antigos, havendo, em alguns casos, sobreposição de copas.

Para subsistência, foram registradas criações como galinhas, patos e frangos, que são vendidos quando existe excedente e demanda. Também se registrou a criação de animais de estimação, como cachorros e gatos. Não há benfeitorias planejadas para abrigar as criações, devido a não haver necessidade pelo clima ser quente, isto é, as criações não sofrerem com a intensificação do frio no inverno, ao contrário dos QAFs do sul do Brasil, a exemplo do estudo de Rondon-Neto et al. (2004) no Paraná, que apontou a existência de abrigos para os animais.

\section{CONCLUSÕES}

Os resultados deste estudo permitiram verificar que: $O$ tempo de formação influencia no número de indivíduos e espécies para a área amostrada; o manejo dos quintais é caracterizado pelo baixo nível de tecnologia empregado e é realizado predominantemente pela mulher.; as espécies destinadas à alimentação (32\%) dominaram a área e as mais frequentes da amostra foram Persea americana, Psidium guajava, Carica papaya e Allium fistulosum; as espécies mais importantes da amostra foram Mangifera indica L., Psidium guajava L., Annona muricata L., Cocos nucifera L., Euterpe oleracea Mart., Carica papaya L., Jatropha gossypiifolia L., Carapa guianensis Aubl. e Citrus limonia Osbeck. Tais espécies podem ser remanejadas para sistemas agroflorestais nos demais lotes do assentamento, otimizar o uso da terra, assegurar a segurança alimentar e maximizar a renda das famílias com a venda do excedente da produção.

\section{REFERÊNCIAS BIBLIOGRÁFICAS}

AGUIAR, J. et al. Reprodução socioeconômica e cultural através do manejo de sistemas agroflorestais por caboclos-ribeirinhos em comunidades do Amazonas. Revista Brasileira de Agroecologia, v. 4, n. 2, 4195-4198, 2009.

ALBUQUERQUE, U. P. et al. Structure and floristics of homegardens in Northeastern Brazil. Journal of Arid Enviroments, v. 62, n. 3, p.491-506, 2005.

ALMEIDA, L. S.; GAMA, J. R. V. Importância socioambiental dos quintais agroflorestais no Assentamento Moju I e II, PA: construindo alternativas sustentáveis na Amazônia. In: JORNADA DE INICIAÇÃO CIENTÍFICA E PESQUISA TECNOLÓGICA DO IESPES, 7., 2010, Santarém. Anais... Santarém, 2010.

BENTES-GAMA, M. M.; GAMA, J. R. V.; TOURINHO, M. M. Huertos caseros en la comunidad ribereña de Villa Cuera, en el municipio de Bragança en el noroeste paraense. Agroforesteria en las Américas, v. 6, n. 4, p. 9-12, 1999.

CASTRO, A. P. de. et al. Os sistemas agroflorestais como alternativa de sustentabilidade em ecossistemas de várzea no Amazonas. Acta Amazonica, v. 39, n. 2, p. 279-288, 2009.

CONSTANTIN, A. A. Quintais agroflorestais na visão dos agricultores de Imaruí-SC. 2005. 120 f. Dissertação (Mestrado em Agroecossistemas)Universidade Federal de Santa Catarina, Florianópolis, 2005. 
COSTA, J. R; MITJA, D. Uso dos recursos vegetais por agricultores familiares de Manacapuru (AM). Acta Amazonica, v. 40, n. 1, p. 49-58, 2010.

DUBOIS, J. C. L. et al. Manual agroflorestal para a Amazônia. Rio de Janeiro: Instituto Rede Brasileira Agroflorestal (REBRAF), v. 1, 1996. $228 \mathrm{p}$.

FERREIRA, T. B.; SABLAYROLLES, M. G. P. Quintais agroflorestais como fontes de saúde: plantas medicinais na comunidade de Vila Franca, Reserva Extrativista Tapajós-Arapiuns, Pará. Revista Brasileira de Agroecologia, v. 4, n. 2, p. 3159-3162, 2009.

FLORENTINO, A. T. N. et al. Contribuição de quintais agroflorestais na conservação de plantas da Caatinga, Município de Caruaru, PE, Brasil. Acta Botanica Brasilica, v. 21, n. 1, p. 37-47, 2007.

GOODE, W. J.; HATT, P. K. Métodos em Pesquisa Social. 3. ed., São Paulo: Cia Editora Nacional, 1969.

IBGE. Manual técnico da vegetação brasileira. Rio de Janeiro: IBGE, 1992. 92 p. (Manuais técnicos de Geociências, 1).

LAKATOS E. M., MARCONI M. A. Fundamentos de Metodologia Científica, v. 4, São Paulo, 2001.

LOURENÇO, J. N. P. et al. Agrobiodiversidade nos Quintais Agroflorestais em Três Assentamentos na Amazônia Central. Revista Brasileira de Agroecologia. v. 4, n. 2, p. 965 - 969, 2009.

LUNZ, A. M. P. Quintais agroflorestais e o cultivo de espécies frutíferas na Amazônia. Revista Brasileira de Agroecologia, v. 2, n. 2, p. 1255-1258, 2007.

NAIR, P. K. P. The enigma of tropical homengardens. Agroflorestry Systems, v. 61, p. 135-152, 2004.

RICO-GRAY, V. et al. Species composition, similarity, and structure of Mayan Homegardens in Tixpeual and Tixcacaltuyub, Yucatan, Mexico. Economic Botany, v. 44, p. 470-487, 1990.

PINTO, E. P. P. et al. Conhecimento popular sobre plantas medicinais em comunidades rurais de mata atlântica - Itacaré, BA. Acta botanica brasilica, v. 20, n. 4, p. 751-762. 2006.

RODRIGUES, T. E. Caracterização dos solos da área do planalto de Belterra, município de Santarém, Estado do Pará. Belém: Embrapa Amazônia Oriental. 2001. 54 p. (Embrapa Amazônia Oriental. Documentos,
$115)$.

RONDON-NETO, R. M. et al. Os quintais agroflorestais do Assentamento Rural Rio da Areia, município de Teixeira Soares, PR. Cerne, v.10, n. 1, p.125-135, 2004.

ROSA, L. S. et al. Os quintais agroflorestais em áreas de agricultores familiares no município de Bragança-PA: composição florística, uso de espécies e divisão de trabalho familiar. Revista Brasileira de Agroecologia, v. 2, n. 2, 2007.

SABLAYROLLES, M. G. P.; ANDRADE, L. Entre sabores e saberes: a importância dos quintais agroflorestais para agricultores ribeirinhos no Tapajós-PA. In: CONGRESSO BRASILEIRO DE SISTEMAS AGROFLORESTAIS, 2009, Brasília, DF. Anais... Brasília, 2009.

SALOMÃO R. de P. et al. As florestas de Belo Monte na grande curva do rio Xingu, Amazônia Oriental. Boletim do Museu Paraense Emílio Goeldi, v. 2, n. 1, p. 57-153, 2007

SANTOS, M. A. C. Levantamento de espécies vegetais úteis das áreas do Sucuriju e região dos Lagos no Amapá. In: Inventário biológico das áreas do Sucuriju e região dos Lagos no Amapá. Documento. MMA/PROBIO/ IEPA, p. 80-106, 2006.

SEMEDO, R. J. C. G.; BARBOSA, R. I.. Árvores frutíferas nos quintais urbanos de Boa Vista, Roraima, Amazônia brasileira. Acta Amazonica, v. 37, n. 4, p. 497-504, 2007.

VELOSO, H. P.; et al.. Classificação da vegetação brasileira adaptada a um sistema universal. Rio de Janeiro: IBGE/Departamento de Recursos Naturais e Estudos Ambientais. 1991. $124 \mathrm{p}$.

VIEIRA, T. A.; ROSA, L. S.; SANTOS, M. M. L. S. Agrobiodiversidade de quintais agroflorestais no município de Bonito, Estado do Pará. Revista de Ciências Agrárias, v. 55, p. 159-166, 2012.

VIEIRA, T. A. Sistemas agroflorestais em áreas de agricultores familiares no município de Igarapé-Açu, Pará: adoção, composição florística e gênero. 2006. 102 f. Dissertação (Mestrado em Ciências Florestais) - Universidade Federal Rural da Amazônia, Belém, 2006.

VÍQUEZ, E. et al. Caracterización del huerto mixto tropical "La Asunción", Masatepe, 
Nicaragua. Agroforesteria en las Américas, n. 2, gardens in Santarém, Pará, Brazil: Linking rural p.5-9, 1994.

with urban. Urban Ecosystems, v. 6, p. 43-65, WINKLERPRINS, A. M. G. A. House-lot 2002. 